\title{
On Interval Observer Design for Continuous-Time LPV Switched Systems
}

\author{
Chaima Zammali, Jeremy Van Gorp ${ }^{a}$, and Tarek Raïssi ${ }^{a}$
}

\begin{abstract}
State estimation for switched systems with time-varying parameters has received a great attention during the past decades. In this paper, a new approach to design an interval observer for this class of systems is proposed. The scheduling vector is described by a convex combination so that the varying parameters belong into polytopes. The considered system is also subject to measurement noise and state disturbances which are supposed to be unknown but bounded. The proposed method guarantees both cooperativity and Input to State Stability (ISS) of the upper and lower observation errors. Sufficient conditions are given in terms of Linear Matrix Inequalities (LMIs) using a common quadratic Lyapunov function. Finally, a numerical example is provided to show the effectiveness of the designed observer.
\end{abstract}

Keywords: interval observer, continuous-time LPV switched systems, polytopic parameter dependence, Lyapunov theory

\section{Introduction}

Over the past few decades, the problem of state estimation for dynamic systems has received a huge attention. Indeed, for economic reasons of feasibility or technological ones, state variables are not always measurable. In the linear case, the state estimation problem has been extensively studied by considering for example Luenberger observers in the deterministic frameworks or Kalman filters in the stochastic settings. In the nonlinear case, state estimation methods are usually based on a system transformation into a canonical form which may be an obstruction in practice. Accordingly, a broad class of nonlinear systems are presented in a Linear Parameter Varying (LPV) form [15, 19, 9]. The main advantage of this strategy is that it allows one to apply several developed frameworks for linear systems. In the literature, many researches have been carried out about LPV systems $[1,10,24]$. However in practice, systems are often subject to exogenous uncertainties (state

\footnotetext{
${ }^{a}$ Conservatoire National des Arts et Métiers (CNAM), Cedric - Lab, HESAM Université, 292 Rue Saint-Martin, 75141 Paris Cedex 03, France, E-mail: chaima.zammali@etu.upmc.fr, E-mail: jeremy.vangorp@lecnam.net, E-mail: tarek.raissi@cnam.fr
} 
disturbance, measurement noise) and endogenous ones (parametric uncertainties). Then, the design of classical observers can be complicated to perform in the presence of uncertainties. The interval observers can be considered as an alternative option to take into account these uncertainties in the estimation procedure.

In a context with unknown but bounded uncertainties, interval estimation consists in evaluating an admissible set for a state vector at each instant of time. In the recent years, many methods for designing interval observers have been proposed for linear and nonlinear systems. In [20, 25, 30], interval observers are designed for linear time-invariant systems and for time-invariant discrete-time systems in [4]. The cooperativity properties are studied with and without state transformation. In [23], interval state estimation is addressed for a class of nonlinear continuoustime systems. In $[3,27,21]$ interval observers for linear time-varying (LTV) systems are introduced where a new approach based on time-varying change of coordinates is provided in order to ensure the cooperativity of the observer error in new coordinates. In [13], two approaches for the transformation of state equations into a cooperative form are presented, a time-invariant transformation for systems with purely real eigenvalues and a time-varying transformation in the case of conjugatecomplex eigenvalues. In [2], interval state estimation is proposed for continuoustime LPV systems under the assumption that the vector of scheduling parameters is not available for measurements. A fault detection procedure and a fault tolerant control based on interval observers are proposed in [31, 14] for LPV systems. A class of LPV systems containing both measured and unmeasured time-varying uncertain parameters is considered in [29] where a polytopic formulation of the estimation problem is developed.

In the literature, an increasing attention has been devoted to switched systems due to their widespread applications in several fields mainly in aerospace, electrical and automotive devices, flight control systems and biological networks [17, 18, 28]. Switched systems are a class of hybrid systems. They involve a finite number of subsystems and a switching rule which governs the switching among them. Interval observers for a class of linear time-invariant switched systems with disturbances is developed in [6, 22]. In [22], the problem of state estimation is investigated for Switched Linear System (SLS). New conditions of cooperativity are given in discrete time instants in order to guarantee the nonnegativity of the estimation errors. As presented for instance in [5], an interval observer is developed for SLS under the assumption that the disturbances and the measurement noise are bounded. The design of interval observer for discrete-time switched systems is also addressed in [7] using a time-varying transformation. As far as interval observers for SLS are studied, interval state estimation of nonlinear switched systems has been also considered. Based on the monotone system theory, an interval observer is designed in [8] to estimate the state of nonlinear switched systems with an average dwell time condition (ADT) using the upper and lower observer variables. In [12], a synchronuous interval observer is designed for switched LPV continuous-time systems using multiple quadratic ISS-Lyapunov functions.

Interval observer design techniques are usually based on the theory of positive systems, which require that in the case of continuous systems the observer 
error state matrix is Metzler. Unfortunately, this assumption is restrictive. Some methods propose a coordinate transformation to cope with the Metzler constraint. However, when systems are affected by time-varying parameters, applying a change of coordinates can constitute an infinite dimensional problem. This motivates the present work, which is devoted to propose a finite dimensional relaxation for both cooperativity and ISS conditions. To the best of the authors knowledge, the design of an interval observer for this class of systems using a polytopic time varying parameters has not been fully studied in the literature.

In this paper, the design of an interval observer for a class of continuous-time LPV switched systems subject to disturbances and time-varying parameters is addressed. The measurement noise and the state disturbances are assumed to be unknown but bounded with known bounds. We assume that the scheduling vector is described by a convex combination so that the varying parameters belong into polytopes. The main advantage of the new proposed approach is that the polytopic varying parameters are explicitly incorporated in the observer design in order to reduce the conservatism. The cooperativity property and the ISS of the estimation errors are ensured. Sufficient ISS conditions are given in terms of LMIs by adopting common quadratic Lyapunov functions.

The outline of this paper is as follows. Some basic preliminaries about continuous-time interval estimation are given in Section 2. The main contributions are developed in Section 3 and Section 4. Simulation results are shown in Section 4. Finally, the paper is concluded in Section 5.

\section{$2 \quad$ Notation and preliminaries}

Throughout this paper, $\mathbb{R}$ and $\mathbb{N}$ denote respectively the sets of real and natural numbers. The symbol $\|\cdot\|_{2}$ denotes the Euclidean norm in $\mathbb{R}^{n}$ and $|\cdot|$ is the componentwise absolute value in $\mathbb{R}^{n}$. For any function $u: \mathbb{R}_{\geq 0} \rightarrow \mathbb{R}^{n}$, we denote by $\|u\|_{\infty}=\sup \left\{\|u(t)\|_{2}, t \geq 0\right\} \leq \infty$ the (essential) supremum norm. In the case of $u$ is bounded, this is the standard $L_{\infty}$ norm. The lower and upper bounds of a variable $x$ are respectively denoted by $\underline{x}$ and $\bar{x}$. We designate by $J_{m}(m \times 1)$ a vector whose elements are equal to 1 and $I_{n}$ is the identity matrix. We denote by $\mathcal{I}=\overline{1, N}, N \in \mathbb{N}$ the set of integers $\{1, \ldots, N\} . P \succ 0, P \succeq 0$ and $P=P^{T}$ mean respectively that $P$ is positive definite, positive semi-definite and symmetric. In the sequel, the following inequalities $\leq, \geq,<$ and $>$ should be interpreted elementwise for vectors as well as for matrices. We recall that a function $\zeta$ is a $\mathcal{K}$-function if it is continuous, strictly increasing and $\zeta(0)=0$; it is a $\mathcal{K}_{\infty}$-function if it is a $\mathcal{K}$-function and also $\zeta(s) \rightarrow \infty$ as $s \rightarrow \infty$; it is a $\mathcal{K} \mathcal{L}$-function if for each fixed $t \geq 0$ the function $\zeta(., t)$ is a $\mathcal{K}$-function and for each fixed $s \geq 0$ it is decreasing to zero as $t \rightarrow \infty$. 


\subsection{Continuous-time switched systems}

A continuous-time switched system can be described by

$$
\left\{\begin{array}{l}
\dot{x}(t)=A_{\sigma(t)} x(t)+B_{\sigma(t)} u(t)+w_{\sigma(t)}(t) \\
y(t)=C x(t)+v(t)
\end{array}, \sigma(t) \in \mathcal{I}\right.
$$

where $x \in \mathbb{R}^{n}, u \in \mathbb{R}^{l}, y \in \mathbb{R}^{m}, w_{\sigma} \in \mathbb{R}^{n}$ and $v \in \mathbb{R}^{m}$ are respectively the state vector, the input and the output, the disturbances and the measurement noise. The switching between subsystems is realized via a switching signal, a piecewise constant function, $\sigma(t): \mathbb{R}_{+} \rightarrow \mathcal{I}$.

\subsection{Cooperative continuous-time linear systems}

Definition 1. A matrix $A \in \mathbb{R}^{n \times n}$ is called Metzler if there exists $\in \in \mathbb{R}^{+}$such that

$$
A+\epsilon I_{n} \geq 0 \text {. }
$$

Lemma 1. Consider the system described by

$$
\dot{x}(t)=A x(t)+u(t)
$$

if $A$ is Metzler, the input $u$ satisfies the inequality $u(t) \geq 0$ and the initial condition $x(0)$ is chosen as $x(0) \geq 0$, then the state $x$ stays nonnegative for all $t \geq 0$. The system (3) is said to be cooperative or nonnegative.

Lemma 2. [11] Consider two vectors $u, v \in \mathbb{R}^{n}$, then

$$
2 u^{T} M v \leq \frac{1}{\varrho} u^{T} M u+\varrho v^{T} M v
$$

holds for any constant $\varrho>0$ and any positive definite matrix $M$.

\subsection{Common Lyapunov functions}

Lemma 3. [16] Let $P \in \mathbb{R}^{n \times n}$ be a symmetric positive definite matrix that satisfies the LMIs

$$
A_{q}{ }^{T} P+P A_{q} \prec 0, \forall q \in \mathcal{I}
$$

then $V(x)=x^{T} P x$ is a Common Quadratic Lyapunov Function for the system (1).

\subsection{Input to State Stability}

Definition 2. [26] The following system

$$
\dot{x}=f(x, u)
$$

is said to be Input to State Stable(ISS) if there exist a $\mathcal{K} \mathcal{L}$-function $\kappa$ and a $\mathcal{K}$ function $\varphi$ such that for each input $u \in L_{\infty}^{m}$ and each $\ell \in \mathbb{R}^{n}$, it holds that

$$
\|x(t, \ell, u)\|_{2} \leq \kappa\left(\|\ell\|_{2}, t\right)+\varphi\left(\|u\|_{\infty}\right), \forall t \geq 0
$$




\section{System description and problem statement}

Consider the following LPV switched system

$$
\left\{\begin{array}{l}
\dot{x}(t)=A_{\sigma(t)}\left(\eta_{q}\right) x(t)+B_{\sigma(t)}\left(\eta_{q}\right) u(t)+w_{\sigma(t)}(t) \\
y(t)=C x(t)+v(t)
\end{array}, \sigma(t) \in \mathcal{I}\right.
$$

where $x \in \mathbb{R}^{n}, u \in \mathbb{R}^{l}, y \in \mathbb{R}^{m}, w_{\sigma} \in \mathbb{R}^{n}$ and $v \in \mathbb{R}^{m}$ are respectively the state vector, the input and the output, the disturbance and the measurement noise. We denote respectively by $A_{\sigma(t)} \in \mathbb{R}^{n \times n}$ and $B_{\sigma(t)} \in \mathbb{R}^{n \times l}$ the state matrices and the input matrices, these matrices are defined with time-varying parameters. The matrix $C \in \mathbb{R}^{m \times n}$ is the output matrix. The switching between the subsystems is realized via a switching signal, a piecewise constant function, $\sigma(t): \mathbb{R}_{+} \rightarrow \mathcal{I}$. In the sequel, the index $q=\sigma(t)$ specifies, at each instant of time, the system currently being followed, $q \in \mathcal{I}=\overline{1, N}, N \in \mathbb{N}, N$ is the number of linear subsystems. For each subsystem, we denote by $\eta_{q}=\left[\eta_{q_{1}}, \ldots, \eta_{q_{r}}\right]^{T}$ the collection of measured time varying parameters, which are constrained in polytopes $E_{q} ; E_{q}$ depends on the active mode. We denote by $\eta_{q}^{(i)}, i=1, \ldots, g$ the vertices of each $E_{q}$. The measurement noise and the state disturbance are supposed to be unknown but bounded.

Assumption 1. We assume that the state matrices $A_{q}\left(\eta_{q}\right)$ and the input $B_{q}\left(\eta_{q}\right)$ depend affinely on $\eta_{q}$

$$
\begin{aligned}
& A_{q}\left(\eta_{q}\right)=A_{q_{0}}+\eta_{q_{1}} A_{q_{1}}+\ldots+\eta_{q_{r}} A_{q_{r}} \\
& B_{q}\left(\eta_{q}\right)=B_{q_{0}}+\eta_{q_{1}} B_{q_{1}}+\ldots+\eta_{q_{r}} B_{q_{r}}
\end{aligned}, q \in \mathcal{I}
$$

Based on this representation and by assuming that the vectors $\eta_{q}$ are measurable, it is worth pointing out that the system matrices are point-valued matrices for the observer implementation but they are overbounded by the convex domain.

As widely known, in several areas of automatic control, one of the main difficulties is how to deal with uncertainties. Therefore, the objective is to design an interval observer for a continuous-time LPV switched system subject to measured time varying parameters, unknown but bounded measurement noise and state disturbance. LMI conditions for cooperativity and ISS of the upper and lower estimation errors shown in the sequel are relaxed thanks to the polytopic shape of the system parameters. They are expressed on the vertices of each polytope in order to avoid any infinite dimensional problem due to the time varying measured parameters. We will consider for that end a particular form of interval observer gains based on the polytopic form of the time-varying parameters.

\section{Main contribution}

In this part, we introduce the following assumptions in order to design an interval observer for continuous-time LPV switched systems. 
Notation 1. We denote by $A_{q}\left(\eta_{q}{ }^{(i)}\right), i=1, \ldots, g$ the vertices of the state matrix $A_{q}\left(\eta_{q}\right)$ of each polytope $E_{q}$ and $L_{q}\left(\eta_{q}{ }^{(i)}\right)$ the vertices of the observer gain.

Assumption 2. The initial state $x(0)$ satisfies $x(0) \leq x(0) \leq \bar{x}(0)$ with known $\underline{x}(0), \bar{x}(0) \in \mathbb{R}^{n}$.

Assumption 3. The measurement noise and the state disturbance are assumed to be unknown but bounded with a priori known bounds such that

$$
\underline{w}_{q} \leq w_{q}(t) \leq \bar{w}_{q},|v(t)| \leq \bar{v} J_{m}
$$

where $\underline{w}_{q}, \bar{w}_{q} \in \mathbb{R}^{n}$ and $\bar{v}$ is a scalar.

Assumption 4. There exist $L_{q}\left(\eta_{q}^{(i)}\right) \in \mathbb{R}^{n \times m}$ such that $A_{q}\left(\eta_{q}^{(i)}\right)-L_{q}\left(\eta_{q}^{(i)}\right) C$ are Metzler for all $\eta_{q}^{(i)} \in E_{q}, i=1, \ldots, g$.

Assumption 5. For all vertices of $E_{q}$ and for all $q \in \mathcal{I}$, the pairs $\left(A_{q}\left(\eta_{q}^{(i)}\right), C\right)$ are detectable.

Assumptions 2-4 are required to ensure the condition of cooperativity. Assumption 3 is common in the literature of interval observers where the state disturbance and the measurement noise are supposed to be unknown but bounded with known bounds. Assumption 5 must be satisfied to build the upper and lower bounds of the continuous state. The detectability of the system is a classical assumption in the field of state estimation. In an approach, this assumption needs to be satisfied for the vertices of all polytopes in order to design the proposed observer.

The aim is to derive two variables $\bar{x}(t)$ and $\underline{x}(t)$ such that $\underline{x}(t) \leq x(t) \leq \bar{x}(t)$, $\forall t \geq 0$ holds despite the state disturbance and the measurement noise provided that Assumption 2 is satisfied. Additionally, the proposed interval observer will guarantee ISS of the estimation errors.

\subsection{Cooperativity of the estimation errors}

In this part, an interval observer for continuous-time LPV switched systems subject to disturbances is considered and the cooperativity property of the observation error is relaxed thanks to the polytopic form of the time-varying parameters.

Theorem 1. Let Assumptions 2-4 and 5 be satisfied then the states of

$$
\left\{\begin{array}{l}
\dot{\bar{x}}=\left(A_{q}\left(\eta_{q}\right)-L_{q}\left(\eta_{q}\right) C\right) \bar{x}+B_{q}\left(\eta_{q}\right) u+\bar{w}_{q}+L_{q}\left(\eta_{q}\right) y+\left|L_{q}\left(\eta_{q}\right)\right| \bar{v} J_{m} \\
\dot{x}=\left(A_{q}\left(\eta_{q}\right)-L_{q}\left(\eta_{q}\right) C\right) \underline{x}+B_{q}\left(\eta_{q}\right) u+\underline{w}_{q}+L_{q}\left(\eta_{q}\right) y-\left|L_{q}\left(\eta_{q}\right)\right| \bar{v} J_{m}
\end{array}, q \in \mathcal{I}\right.
$$

and (6) satisfies $\underline{x}(t) \leq x(t) \leq \bar{x}(t)$ where the observer gain $L_{q}\left(\eta_{q}\right)$ has an affine form given by

$$
L_{q}\left(\eta_{q}\right)=L_{q_{0}}+\eta_{q_{1}} L_{q_{1}}+\ldots+\eta_{q_{r}} L_{q_{r}}
$$

and $L_{q_{j}} \in \mathbb{R}^{n \times m}, j=0,1, \ldots, r$, are constant matrices. 
Proof. Let $T_{q}\left(\eta_{q}\right)=A_{q}\left(\eta_{q}\right)-L_{q}\left(\eta_{q}\right) C . A_{q}\left(\eta_{q}\right)$ and $L_{q}\left(\eta_{q}\right)$ depend affinely of $\eta_{q}$. Consequently $T_{q}$ can be written as a convex combination form

$$
\begin{aligned}
T_{q}\left(\eta_{q}\right) & =\lambda_{1} T_{q}\left(\eta_{q}{ }^{(1)}\right)+\ldots+\lambda_{g} T_{q}\left(\eta_{q}^{(g)}\right) \\
& =\lambda_{1}\left(A_{q}\left(\eta_{q}{ }^{(1)}\right)-L_{q}\left(\eta_{q}^{(1)}\right) C\right)+\ldots+\lambda_{g}\left(A_{q}\left(\eta_{q}{ }^{(g)}\right)-L_{q}\left(\eta_{q}{ }^{(g)}\right) C\right) \\
& =\sum_{i=1}^{g} \lambda_{i}\left(A_{q}\left(\eta_{q}^{(i)}\right)-L_{q}\left(\eta_{q}^{(i)}\right) C\right)
\end{aligned}
$$

with $\lambda_{i} \geq 0$ and $\lambda_{1}+\ldots+\lambda_{g}=1$

First of all, let $\bar{e}(t)=\bar{x}-x$ and $\underline{e}(t)=x-\underline{x}$ be the upper and the lower observation errors. From (6), (9) and (11), the dynamics of the interval estimation errors are given by

$$
\begin{aligned}
\dot{\bar{e}}(t) & =\dot{\bar{x}}-\dot{x} \\
& =\left(A_{q}\left(\eta_{q}\right)-L_{q}\left(\eta_{q}\right) C\right) \bar{x}+B_{q}\left(\eta_{q}\right) u+\bar{w}_{q}+L_{q}\left(\eta_{q}\right) y+\left|L_{q}\left(\eta_{q}\right)\right| \bar{v} J_{m}- \\
& \quad\left(A_{q}\left(\eta_{q}\right) x+B_{q}\left(\eta_{q}\right) u+w_{q}\right) \\
= & \left(A_{q}\left(\eta_{q}\right)-L_{q}\left(\eta_{q}\right)\left(\eta_{q}\right) C\right)(\bar{x}-x)+\bar{w}_{q}-w_{q}+L_{q}\left(\eta_{q}\right) v+\left|L_{q}\left(\eta_{q}\right)\right| \bar{v} J_{m} \\
= & \left(A_{q}\left(\eta_{q}\right)-L_{q}\left(\eta_{q}\right) C\right) \bar{e}+\bar{w}_{q}-w_{q}+L_{q}\left(\eta_{q}\right) v+\left|L_{q}\left(\eta_{q}\right)\right| \bar{v} J_{m} \\
= & \sum_{i=1}^{g} \lambda_{i}\left(A_{q}\left(\eta_{q}{ }^{(i)}\right)-L_{q}\left(\eta_{q}{ }^{(i)}\right) C\right) \bar{e}+\bar{\chi}_{q}
\end{aligned}
$$

where

$$
\bar{\chi}_{q}=\bar{w}_{q}-w_{q}+L_{q}\left(\eta_{q}\right) v+\left|L_{q}\left(\eta_{q}\right)\right| \bar{v} J_{m} .
$$

Similarly, we have

$$
\underline{\dot{e}}(t)=\sum_{i=1}^{g} \lambda_{i}\left(A_{q}\left(\eta_{q}{ }^{(i)}\right)-L_{q}\left(\eta_{q}{ }^{(i)}\right) C\right) \underline{e}+\underline{\chi}_{q}
$$

where

$$
\underline{\chi}_{q}=w_{q}-\underline{w}_{q}-L_{q}\left(\eta_{q}\right) v+\left|L_{q}\left(\eta_{q}\right)\right| \bar{v} J_{m}
$$

According to Assumption 3, it follows that $\bar{\chi}_{q} \geq 0$ and $\underline{\chi}_{q} \geq 0$. The requirement on the cooperativity property of the matrix $\left(A_{q}\left(\eta_{q}\right)-L_{q}\left(\eta_{q}\right) C\right)$ is relaxed thanks to the polytopic form of the time-varying parameters. Based on (11), to show that $\left(A_{q}\left(\eta_{q}\right)-L_{q}\left(\eta_{q}\right) C\right)$ is Metzler is reduced to show that all matrices $A_{q}\left(\eta_{q}{ }^{(i)}\right)-$ $L_{q}\left(\eta_{q}{ }^{(i)}\right) C$, for $i=1, \ldots, g$, are Metzler. To that end, based on Definition 1, the Metzler property is satisfied for all $\eta_{q}^{(i)}$ :

$$
A_{q}\left(\eta_{q}^{(i)}\right)-L_{q}\left(\eta_{q}{ }^{(i)}\right) C+\epsilon I_{n} \geq 0, \forall q \in \mathcal{I}, \epsilon \in \mathbb{R}^{+} .
$$

It follows that, from Assumption 4, the dynamics of the upper and lower errors are cooperative. Accordingly to Lemma $1, \underline{x}(t) \leq x(t) \leq \bar{x}(t)$ provided that $\underline{x}(0) \leq x(0) \leq \bar{x}(0)$. 


\subsection{Input to State Stability of the estimation errors}

In order to check the boundedness of $\underline{e}$ and $\bar{e}$, we consider a common Lyapunov function for the estimation errors. In the following theorem, ISS conditions are given in terms of LMIs defined at the vertices of each polytope. Indeed, by assuming that the scheduling vector is described by a convex combination and that its timevarying parameters belong to polytopes, LMIs and the proposed observer gains $L_{q}\left(\eta_{q}\right)$ are formulated in terms of the vertices of these polytopes.

Theorem 2. Consider the continuous-time LPV switched system (6), where $A_{q}\left(\eta_{q}\right)$ and $B_{q}\left(\eta_{q}\right)$ are affine matrices on $\eta_{q}$ and let Assumption 4 hold. If there exist a diagonal $P \succ 0 \in \mathbb{R}^{n \times n}$, constant matrices $Q_{q_{0}}, \ldots, Q_{q_{r}}$ and scalars $\varrho_{q}>0, \forall q \in \mathcal{I}$ such that

$$
A_{q}\left(\eta_{q}^{(i)}\right)^{T} P+P A_{q}\left(\eta_{q}^{(i)}\right)-\left(C^{T} Q_{q}\left(\eta_{q}^{(i)}\right)^{T}+Q_{q}\left(\eta_{q}^{(i)}\right) C\right)+\frac{3}{\varrho_{q}} P \prec 0, \forall q \in \mathcal{I}
$$

where $Q_{q}\left(\eta_{q}^{(i)}\right)$ are affine matrices of $\eta_{q}^{(i)}$ given by

$$
Q_{q}\left(\eta_{q}^{(i)}\right)=Q_{q_{0}}+\eta_{q_{1}}^{(i)} Q_{q_{1}}+\ldots+\eta_{q_{r}}^{(i)} Q_{q_{r}}
$$

with $Q_{q_{j}} \in \mathbb{R}^{n \times m}, j=0,1, \ldots, r$ are constant matrices, then the observer gains $L_{q_{j}}, j=0,1, \ldots, r$ are obtained as

$$
L_{q_{j}}=P^{-1} Q_{q_{j}}
$$

and the states $\bar{x}, \underline{x}$ are bounded.

Proof. For the stability analysis, ISS of the interval observer is ensured by using a common Lyapunov function to the upper and lower estimation errors. Let us consider the common Lyapunov function applied, first of all, to the upper estimation error $V(\bar{e})=\bar{e}(t)^{T} P \bar{e}(t)$ with $P=P^{T} \succ 0$.

Based on (12), the derivative of $V$ is given by

$$
\begin{aligned}
\dot{V}(\bar{e})= & \dot{\bar{e}}^{T} P \bar{e}+\bar{e}^{T} P \dot{\bar{e}} \\
= & \left(\left(A_{q}\left(\eta_{q}\right)-L_{q}\left(\eta_{q}\right) C\right) \bar{e}+\bar{w}_{q}-w_{q}+L_{q}\left(\eta_{q}\right) v+\left|L_{q}\left(\eta_{q}\right)\right| \bar{v} J_{m}\right)^{T} P \bar{e}+ \\
& \bar{e}^{T} P\left(\left(A_{q}\left(\eta_{q}\right)-L_{q}\left(\eta_{q}\right) C\right) \bar{e}+\bar{w}_{q}-w_{q}+L_{q}\left(\eta_{q}\right) v+\left|L_{q}\left(\eta_{q}\right)\right| \bar{v} J_{m}\right) \\
= & \bar{e}^{T}\left(\left(A_{q}\left(\eta_{q}\right)-L_{q}\left(\eta_{q}\right) C\right)^{T} P+P\left(A_{q}\left(\eta_{q}\right)-L_{q}\left(\eta_{q}\right) C\right)\right) \bar{e}+ \\
& \bar{w}_{q}^{T} P \bar{e}-w_{q}^{T} P \bar{e}+\left(L_{q}\left(\eta_{q}\right) v\right)^{T} P \bar{e}+\left(\left|L_{q}\left(\eta_{q}\right)\right| \bar{v} J_{m}\right)^{T} P \bar{e}+ \\
& \bar{e}^{T} P \bar{w}_{q}-\bar{e}^{T} P w_{q}+\bar{e}^{T} P\left(L_{q}\left(\eta_{q}\right) v\right)+\bar{e}^{T} P\left|L_{q}\left(\eta_{q}\right)\right| \bar{v} J_{m} \\
= & \bar{e}^{T}\left(\left(A_{q}\left(\eta_{q}\right)-L_{q}\left(\eta_{q}\right) C\right)^{T} P+P\left(A_{q}\left(\eta_{q}\right)-L_{q}\left(\eta_{q}\right) C\right)\right) \bar{e}- \\
& 2 \bar{e}^{T} P w_{q}+2 \bar{e}^{T} P L_{q}\left(\eta_{q}\right) v+2 \bar{e}^{T} P \bar{w}_{q}+2 \bar{e}^{T} P\left|L_{q}\left(\eta_{q}\right)\right| \bar{v} J_{m} .
\end{aligned}
$$

Based on Lemma 2, we have

$$
2 \bar{e}^{T} P\left(\bar{w}_{q}-w_{q}\right) \leq \frac{1}{\varrho_{q}} \bar{e}^{T} P \bar{e}+\varrho_{q}\left(\bar{w}_{q}-w_{q}\right)^{T} P\left(\bar{w}_{q}-w_{q}\right)
$$




$$
\begin{gathered}
2 \bar{e}^{T} P L_{q}\left(\eta_{q}\right) v \leq \frac{1}{\varrho_{q}} \bar{e}^{T} P \bar{e}+\varrho_{q} v^{T} L_{q}\left(\eta_{q}\right)^{T} P L_{q}\left(\eta_{q}\right) v \\
2 \bar{e}^{T} P\left|L_{q}\left(\eta_{q}\right)\right| \bar{v} J_{m} \leq \frac{1}{\varrho_{q}} \bar{e}^{T} P \bar{e}+\varrho_{q} J_{m}{ }^{T} \bar{v}\left|L_{q}\left(\eta_{q}\right)\right|^{T} P\left|L_{q}\left(\eta_{q}\right)\right| \bar{v} J_{m},
\end{gathered}
$$

then, the derivative of $V$ satisfies

$$
\dot{V}(\bar{e}) \leq \bar{e}^{T} B_{1} \bar{e}+C_{1}
$$

where

$$
\begin{aligned}
B_{1} & =\left(A_{q}\left(\eta_{q}\right)-L_{q}\left(\eta_{q}\right) C\right)^{T} P+P\left(A_{q}\left(\eta_{q}\right)-L_{q}\left(\eta_{q}\right) C\right)+\frac{3}{\varrho_{q}} P \\
& =\sum_{i=1}^{g} \lambda_{i}\left(A_{q}\left(\eta_{q}{ }^{(i)}\right)^{T} P+P A_{q}\left(\eta_{q}{ }^{(i)}\right)-C^{T} Q_{q}\left(\eta_{q}{ }^{(i)}\right)^{T}-Q_{q}\left(\eta_{q}{ }^{(i)}\right) C\right)+\frac{3}{\varrho_{q}} P
\end{aligned}
$$

where $Q_{q}\left(\eta_{q}^{(i)}\right)=P L_{q}\left(\eta_{q}^{(i)}\right)$, and

$$
\begin{gathered}
C_{1}=\varrho_{q}\left(\bar{w}_{q}-w_{q}\right)^{T} P\left(\bar{w}_{q}-w_{q}\right)+\varrho_{q} v^{T} L_{q}\left(\eta_{q}\right)^{T} P L_{q} v+ \\
\varrho_{q} J_{m}{ }^{T} \bar{v}\left|L_{q}\left(\eta_{q}\right)\right|^{T} P\left|L_{q}\left(\eta_{q}\right)\right| \bar{v} J_{m} .
\end{gathered}
$$

Based on similar arguments, the derivate of the common Lyapunov function for the lower estimation error is written as follow

$$
\begin{aligned}
\dot{V}(\underline{e})= & \underline{e}^{T}\left(\left(A_{q}\left(\eta_{q}\right)-L_{q}\left(\eta_{q}\right) C\right)^{T} P+P\left(A_{q}\left(\eta_{q}\right)-L_{q}\left(\eta_{q}\right) C\right)\right) \underline{e}+ \\
& 2 \underline{e}^{T} P w_{q}-2 \underline{e}^{T} P \underline{w}_{q}-2 \underline{e}^{T} P L_{q}\left(\eta_{q}\right) v+2 \underline{e}^{T} P\left|L_{q}\left(\eta_{q}\right)\right| \underline{v} J_{m} \\
\leq & \underline{e}^{T} B_{1} \underline{e}+C_{2}
\end{aligned}
$$

where

$$
\begin{gathered}
C_{2}=\varrho_{q}\left(w_{q}-\underline{w}_{q}\right)^{T} P\left(w_{q}-\underline{w}_{q}\right)+\varrho_{q} v^{T} L_{q}\left(\eta_{q}\right)^{T} P L_{q}\left(\eta_{q}\right) v+ \\
\varrho_{q} J_{m}{ }^{T} \bar{v}\left|L_{q}\left(\eta_{q}\right)\right|^{T} P\left|L_{q}\left(\eta_{q}\right)\right| \bar{v} J_{m} .
\end{gathered}
$$

From (16), we note that $B_{1} \prec 0$. Under the Assumption 3, the uncertainties $w_{q}$ and $v$ are bounded, $C_{1}$ and $C_{2}$ are also bounded. Based on Definition 2, the system (9) is ISS stable and the upper and lower estimation errors are bounded.

\section{$5 \quad$ Numerical example}

In this section, a numerical example is considered to illustrate the performance of the proposed interval observer for a continuous-time LPV switched system. 


\subsection{LPV switched modeling}

Let us consider the continuous-time LPV switched system (6) defined with three subsystems, $N=3$. Based on the representation (7), state matrices, input matrices and the output matrix are chosen as

$$
\begin{gathered}
A_{1_{0}}=\left[\begin{array}{ll}
-2 & 6 \\
-1 & 5
\end{array}\right], A_{1_{1}}=\left[\begin{array}{cc}
-1 & 2 \\
-1 & -1
\end{array}\right], A_{1_{2}}=\left[\begin{array}{cc}
2 & -1 \\
-1 & -2
\end{array}\right], \\
B_{1_{0}}=\left[\begin{array}{cc}
-1 & 1 \\
1 & 0
\end{array}\right], B_{1_{1}}=\left[\begin{array}{cc}
-1 & 1 \\
2 & 3
\end{array}\right], B_{1_{2}}=\left[\begin{array}{ll}
2 & 1 \\
1 & 2
\end{array}\right], \\
A_{2_{0}}=\left[\begin{array}{cc}
-3 & 4 \\
-5 & -2
\end{array}\right], A_{2_{1}}=\left[\begin{array}{cc}
-1 & -3 \\
2 & -2
\end{array}\right], A_{2_{2}}=\left[\begin{array}{cc}
1 & 2 \\
-1 & -1
\end{array}\right], \\
B_{2_{0}}=\left[\begin{array}{cc}
-1 & 2 \\
1.5 & 0
\end{array}\right], B_{2_{1}}=\left[\begin{array}{cc}
-1 & 2 \\
1 & 1
\end{array}\right], B_{2_{2}}=\left[\begin{array}{cc}
2 & 2 \\
3 & 2
\end{array}\right], \\
A_{3_{0}}=\left[\begin{array}{cc}
-3.5 & 5 \\
-1 & -2
\end{array}\right], A_{3_{1}}=\left[\begin{array}{cc}
-3 & 2 \\
1 & 1
\end{array}\right], A_{3_{2}}=\left[\begin{array}{cc}
1 & -2 \\
-3 & -3
\end{array}\right], \\
B_{3_{0}}=\left[\begin{array}{cc}
-2 & 1.5 \\
1 & 0
\end{array}\right], B_{3_{1}}=\left[\begin{array}{cc}
-1 & 2 \\
3 & 2
\end{array}\right], B_{3_{2}}=\left[\begin{array}{cc}
1 & 3 \\
3 & 1
\end{array}\right], \\
C=\left[\begin{array}{cc}
1 & -1
\end{array}\right] .
\end{gathered}
$$

For simulation, $x=\left[x_{1}, x_{2}\right]^{T} \in \mathbb{R}^{2}$ is the state, $y \in \mathbb{R}$ is the output and $u=[1,1]^{T} \in$ $\mathbb{R}^{2}$ is the known input. It is assumed in this example that, $w_{q}(t) \in \mathbb{R}^{2}, q=1,2,3$, the disturbance vector is supposed to be bounded. To that end, $w_{q}(t)$ is chosen as follow: $w_{1}(t)=[0.009,0.001]^{T} \cos (5 t), w_{2}(t)=[0.002,0.003]^{T} \cos (2 t), w_{3}(t)=$ $[0.003,0.004]^{T} \cos (3 t) . v(t)$ represents the measurement noise: $v(t)=0.07 \sin (t)$. The state initial conditions are set as $x(0)=[0,0]^{T}$ such that: $\underline{x}(0) \leq x(0) \leq \bar{x}(0)$. The measured parameter vector $\eta_{q} \in \mathbb{R}^{2}, q=1,2,3$, is given by

$$
\begin{gathered}
\eta_{1}(t)=\left[\begin{array}{c}
|\sin (2 t)|+4 \\
|\cos (t)|+4
\end{array}\right], \eta_{2}(t)=\left[\begin{array}{c}
|2 \cos (0.2 t)|+4 \\
|2 \cos (3 t)|+4
\end{array}\right], \\
\eta_{3}(t)=\left[\begin{array}{c}
|3 \cos (0.2 t)|+4 \\
|3 \cos (t)|+4
\end{array}\right] .
\end{gathered}
$$

The vertices of the polytope can be easily deduced from these expressions.

\subsection{Simulation results}

The numerical simulation was carried out by using Matlab optimization tools (Yalmip/Sedumi). Solving the LMIs conditions, one feasible solution is given by

$$
P=\left[\begin{array}{cc}
2.08 & 0 \\
0 & 5.03
\end{array}\right]
$$


The observer gains $L_{q_{j}}$, for $q=1,2,3$ and $j=0,1,2$ are computed using the expression (18). Thus, we have

$$
\begin{aligned}
& L_{1_{0}}=\left[\begin{array}{ll}
125 & -49.8
\end{array}\right]^{T}, L_{1_{1}}=\left[\begin{array}{ll}
-46.5 & 25.1
\end{array}\right]^{T}, L_{1_{2}}=\left[\begin{array}{ll}
50.2 & -23.5
\end{array}\right]^{T}, \\
& L_{2_{0}}=\left[\begin{array}{ll}
129 & -53.4
\end{array}\right]^{T}, L_{2_{1}}=\left[\begin{array}{ll}
-24.1 & -12.8
\end{array}\right]^{T}, L_{2_{2}}=\left[\begin{array}{ll}
24.9 & -10.5
\end{array}\right]^{T}, \\
& L_{3_{0}}=\left[\begin{array}{ll}
128 & -54.2
\end{array}\right]^{T}, L_{3_{1}}=\left[\begin{array}{ll}
-15.2 & 10.1
\end{array}\right]^{T}, L_{3_{2}}=\left[\begin{array}{ll}
17.6 & -9.41
\end{array}\right]^{T} .
\end{aligned}
$$

Remark 1. The LMI (16) is formulated with the aim to find observer gains ensuring the interval observer stability. In others words, the observer gains which are functions of $\eta_{q}$ will decide only the stability of $\underline{e}$ and $\bar{e}$. In our example, the goal is to check the boundedness and the positivity of $\underline{e}$ and $\bar{e}$. Based on the proposed interval observer, the disturbance and measurement noise have an influence on the estimation errors $\underline{e}$ and $\bar{e}$. The optimization problem can be reformulated in order to improve its accuracy by providing a minimization of the estimation errors. Hence, the observer gains could ensure not only the stability requirement but also the tightness of the interval width. An optimization with respect to $H_{\infty}$ performance specifications could be introduced to enhance the robustness of the observation error intervals. In other words, one will focus on computing observer gains $L_{q}\left(\eta_{q}\right)$ minimizing the following cost function

$$
\begin{gathered}
\min _{L_{q}} \gamma^{2}, q=1, \ldots, N \\
\text { subject to } \frac{\|\bar{e}\|_{2}^{2}}{\|\bar{\chi}\|_{2}^{2}} \leq \gamma^{2}
\end{gathered}
$$

with $\bar{e}$ is the upper bound of the estimation error and $\bar{\chi}$ is an input which takes into account the bound of the disturbance and noises. $\gamma$ is a positive real number. Accordingly, the effect of the known bound of the uncertainties $\bar{\chi}=\bar{w}_{q}-w_{q}+$ $L_{q}\left(\eta_{q}\right) v+\left|L_{q}\left(\eta_{q}\right)\right| \bar{v} J_{m}$ on the upper bound of estimation error, $\bar{e}$ can be optimized by the observer gain matrices $L_{q}\left(\eta_{q}\right)$. However, this optimization is not considered in this paper.

Under the switching sequence shown in Fig. 1, the simulation results of the interval observer are depicted in Fig. 2. Figure 3 represents an enlarged view of the results in Fig. 2. It is worth noting that the state $x$ is between the lower and upper bounds $\underline{x}$ and $\bar{x}$. In order to highlight the performance of the proposed interval observer, one can remark that the errors $\underline{e}$ and $\bar{e}$ stay bounded and positive. The simulation results of the evolutions of the estimation errors are presented in Fig. 4 and Fig. 5. 


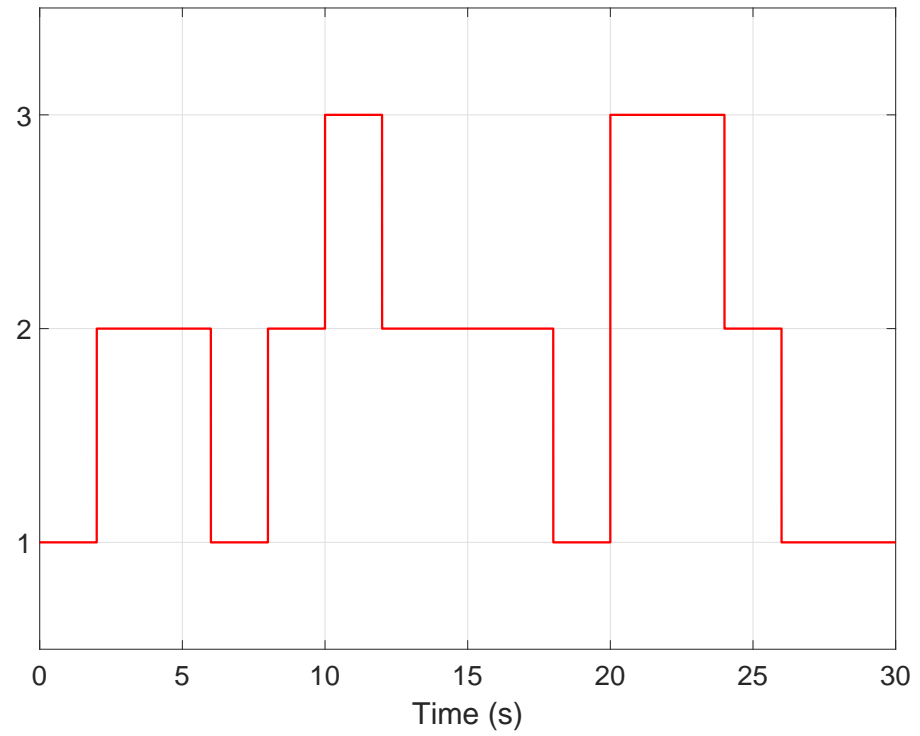

Figure 1: Evolution of the switching signal
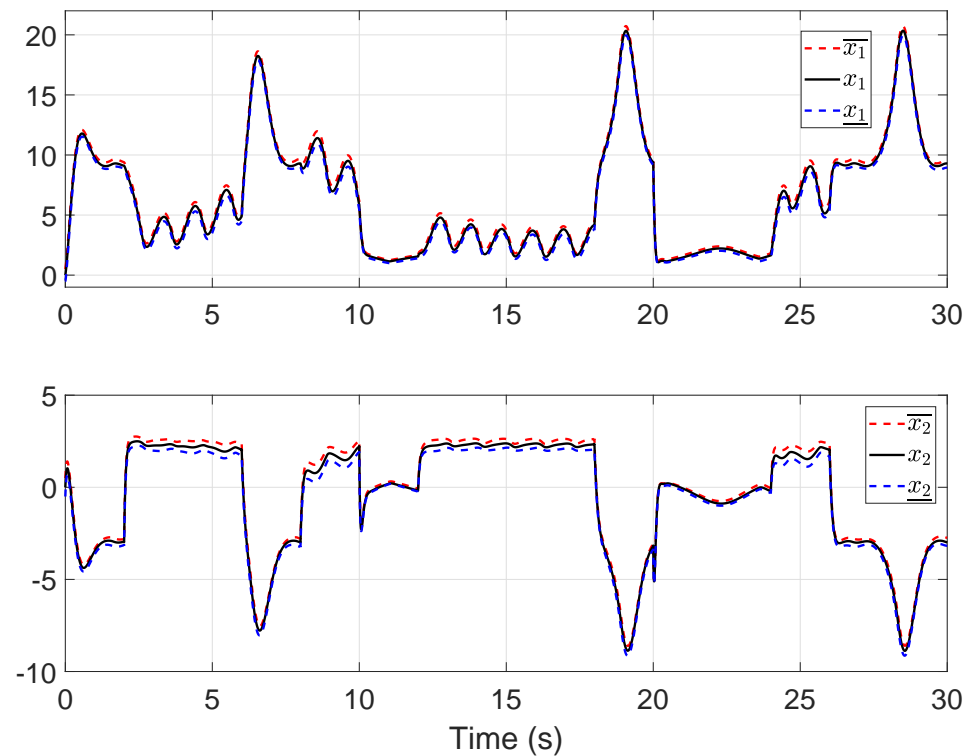

Figure 2: Evolution of the state $x$ and the estimated upper and lower bounds $\bar{x}$ and $\underline{x}$. 

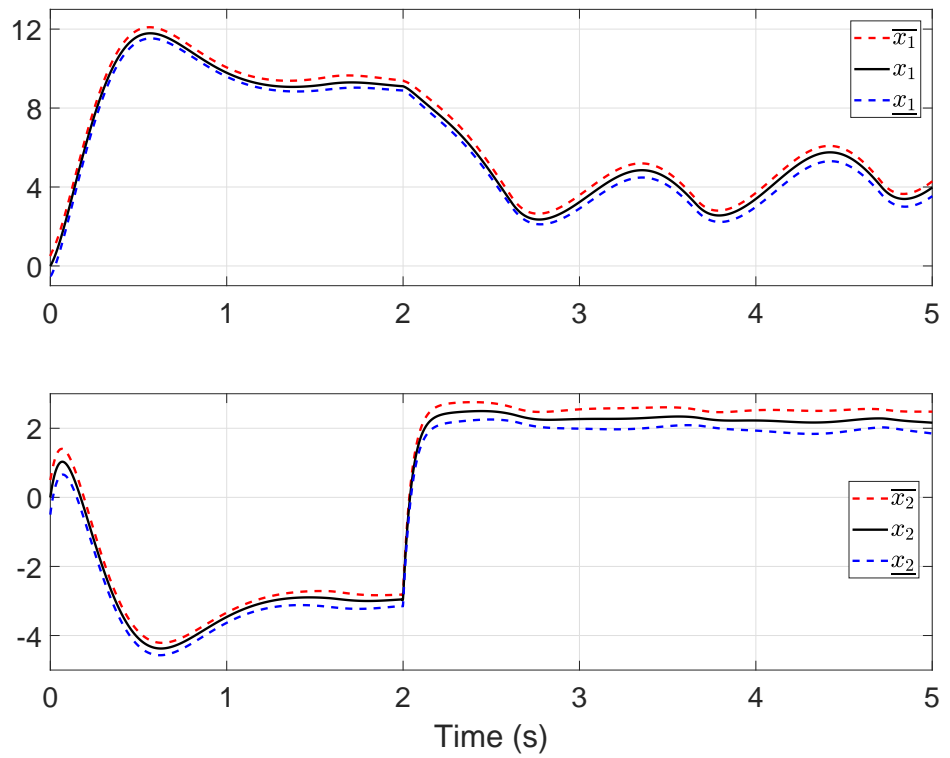

Figure 3: Evolution of the state $x$ and the estimated upper and lower bounds $\bar{x}$ and $\underline{x}(\mathrm{ZOOM})$.
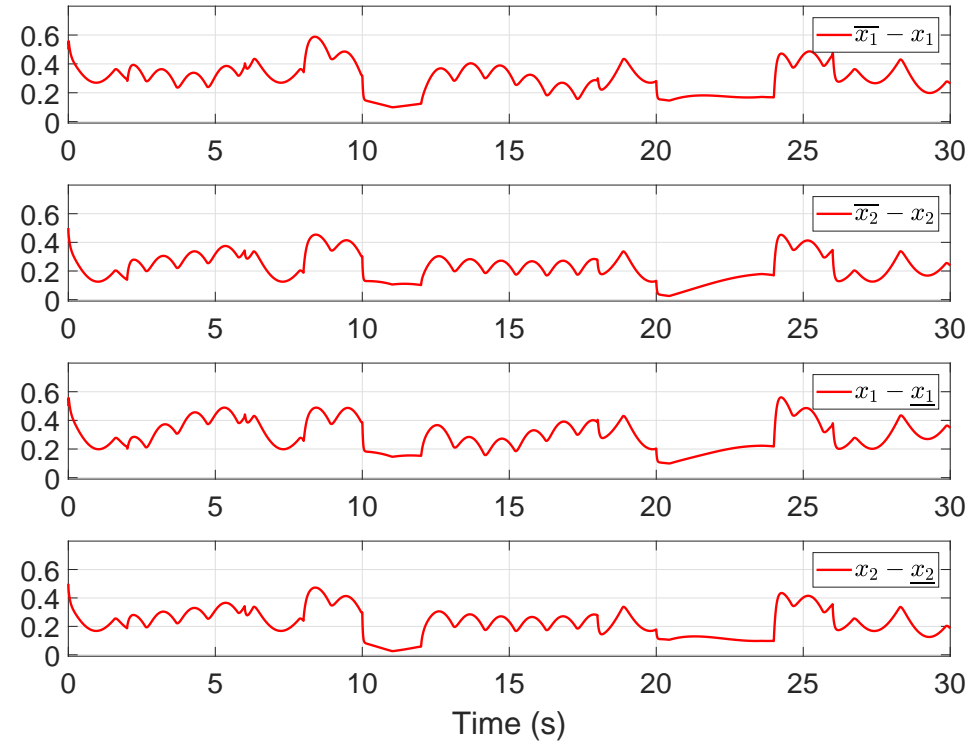

Figure 4: Evolution of the estimation errors 

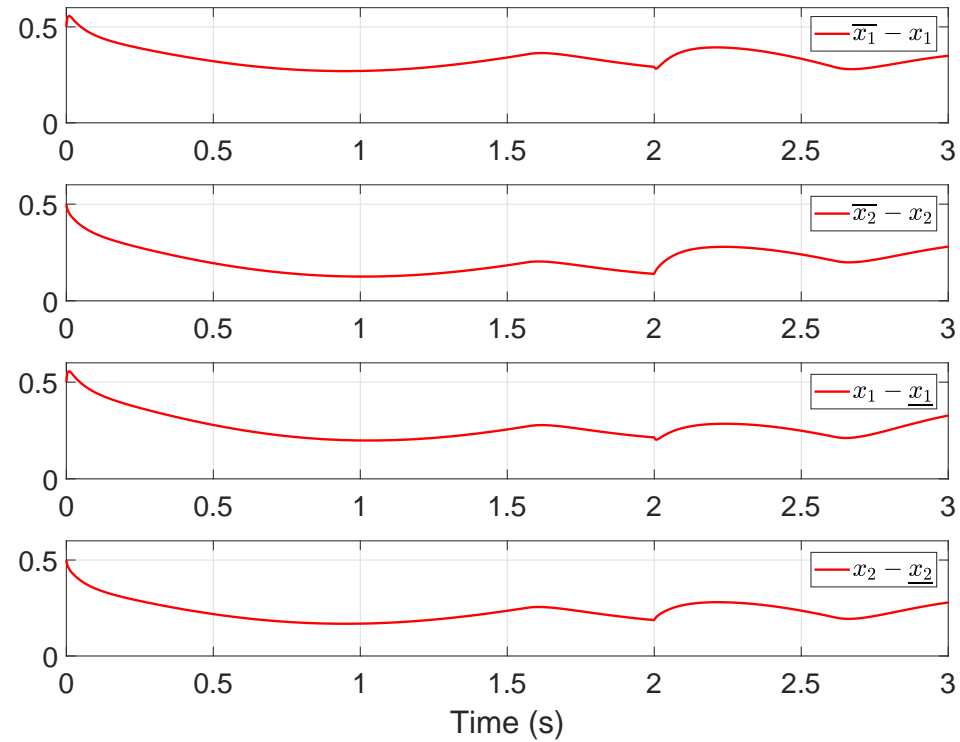

Figure 5: Evolution of the estimation errors (ZOOM)

\section{Conclusions and outlook on future work}

In this paper, an interval observer approach is developed for continuous-time LPV switched systems with polytopic time-varying parameters which are supposed to be measured. Under the assumption that the measurement noise and the state disturbance are unknown but bounded, cooperativity and input to state stability of the upper and lower bounds of the errors are provided. The key advantage of this contribution is the reduction of the conservatism thanks to the polytopic form of the time-varying parameters. Simulation results are given to illustrate the efficiency of the approach. For future works, extensions of these results with unmeasured scheduling vector and the relaxation of Assumption 4 by finding a change of coordinates can be expected.

\section{References}

[1] Anstett, Floriane, Millérioux, Gilles, and Bloch, Gérard. Polytopic observer design for LPV systems based on minimal convex polytope finding. Journal of Algorithms \& Computational Technology, 3(1):23-43, 2009. DOI: $10.1260 / 174830109787186569$. 
[2] Chebotarev, Stanislav, Efimov, Denis, Raïssi, Tarek, and Zolghadri, Ali. Interval observers for continuous-time LPV systems with 11/12 performance. Automatica, 58:82-89, 2015. DOI: 10.1016/j.automatica.2015.05.009.

[3] Efimov, Denis, Perruquetti, Wilfrid, Raïssi, Tarek, and Zolghadri, Ali. Interval observers for time-varying discrete-time systems. IEEE Transactions on Automatic Control, 58(12):3218-3224, 2013. DOI: 10.1109/tac. 2013.2263936.

[4] Efimov, Denis, Perruquetti, Wilfrid, Raïssi, Tarek, and Zolghadri, Ali. On interval observer design for time-invariant discrete-time systems. In European Control Conference (ECC), pages 2651-2656. IEEE, July 2013, Zurich, Switzerland. DOI: 10.23919/ecc.2013.6669108.

[5] Ethabet, Haifa, Rabehi, Djahid, Efimov, Denis, and Raïssi, Tarek. Interval estimation for continuous-time switched linear systems. Automatica, 90:230238, 2018. DOI: 10.1016/j.automatica.2017.12.035.

[6] Ethabet, Haifa, Raïssi, Tarek, Amairi, Messaoud, and Aoun, Mohamed. Interval observers design for continuous-time linear switched systems. IFACPapersOnLine, 50(1):6259-6264, July 2017, Toulouse, France. DOI: $10.1016 / \mathrm{j}$. ifacol.2017.08.853.

[7] Guo, Shenghui and Zhu, Fanglai. Interval observer design for discrete-time switched system. IFAC-PapersOnLine, 50(1):5073-5078, July 2017, Toulouse, France. DOI: $10.1016 / j$.ifacol.2017.08.957.

[8] He, Zhongwei and Xie, Wei. Interval state observer for nonlinear switched systems with average dwell time. In 34th Chinese Control Conference (CCC), pages 2285-2288, July 2015, Hangzhou, China. DOI: 10.1109/chicc.2015.7259989.

[9] Hecker, Simon and Varga, Andras. Generalized lft-based representation of parametric uncertain models. European Journal of Control, 10(4):326-337, 2004. DOI: $10.23919 /$ ecc. 2003.7085049.

[10] Heemels, WP Maurice H, Daafouz, Jamal, and Millerioux, Gilles. Observerbased control of discrete-time LPV systems with uncertain parameters. IEEE Transactions on Automatic Control, 55(9):2130-2135, 2010. DOI: 10.1109/tac.2010.2051072.

[11] Hespanha, Joao P and Morse, A Stephen. Stability of switched systems with average dwell-time. In Proceedings of the 38th IEEE Conference on Decision and Control $(C D C)$, pages 2655-2660, August 2002, Phoenix, AZ, USA, USA. DOI: $10.1109 /$ cdc.1999.831330.

[12] Ifqir, Sara, Ait-Oufroukh, Naima, Ichalal, Dalil, and Mammar, Saïd. Synchronous interval observer design for switched LPV systems using multiple 
quadratic iss-lyapunov functions. In 25th Mediterranean Conference on Control and Automation (MED), pages 388-393. IEEE, July 2017, Valletta, Malta. DOI: $10.1109 / \mathrm{med} .2017 .7984149$.

[13] Kersten, Julia, Rauh, Andreas, and Aschemann, Harald. State-space transformations of uncertain systems with purely real and conjugate-complex eigenvalues into a cooperative form. In 2018 23rd International Conference on Methods $\& 3$ Models in Automation \& Robotics (MMAR), pages 797-802. IEEE, August 2018, Miedzyzdroje, Poland. DOI: 10.1109/mmar.2018.8486085.

[14] Lamouchi, Rihab, Raïssi, Tarek, Amairi, Messaoud, and Aoun, Mohamed. Interval observer framework for fault-tolerant control of linear parametervarying systems. International Journal of Control, 91(3):524-533, 2018. DOI: 10.1080/00207179.2017.1286042.

[15] Lee, Lawton Hubert. Identification and robust control of linear parametervarying systems. PhD Thesis, 1997. University of California, Berkeley.

[16] Liberzon, Daniel. Switching in systems and control. Springer Science \& Business Media, 2003. DOI: 10.1007/978-1-4612-0017-8.

[17] Liu, Jianxing, Vazquez, Sergio, Wu, Ligang, Marquez, Abraham, Gao, Huijun, and Franquelo, Leopoldo G. Extended state observer-based sliding-mode control for three-phase power converters. IEEE Transactions on Industrial Electronics, 64(1):22-31, 2017. DOI: 10.1109/tie.2016.2610400.

[18] Lu, Bei, Wu, Fen, and Kim, SungWan. Switching LPV control of an f-16 aircraft via controller state reset. IEEE Transactions on Control Systems Technology, 14(2):267-277, 2006. DOI: 10.1109/tcst.2005.863656.

[19] Marcos, Andrés and Balas, Gary J. Development of linear-parameter-varying models for aircraft. Journal of Guidance, Control, and Dynamics, 27(2):218228, 2004. DOI: 10.2514/1.9165.

[20] Mazenc, Frédéric and Bernard, Olivier. Interval observers for linear timeinvariant systems with disturbances. Automatica, 47(1):140-147, 2011. DOI: 10.1016/j.automatica.2010.10.019.

[21] Mazenc, Frédéric, Dinh, Thach Ngoc, and Niculescu, Silviu Iulian. Interval observers for discrete-time systems. International Journal of Robust and Nonlinear Control, 24(17):2867-2890, 2014. DOI: 10.1002/rnc.3030.

[22] Rabehi, Djahid, Efimov, Denis, and Richard, Jean-Pierre. Interval estimation for linear switched system. IFAC-PapersOnLine, 50(1):6265-6270, July 2017, Toulouse, France. DOI: 10.1016/j.ifacol.2017.08.855.

[23] Raïssi, Tarek, Efimov, Denis, and Zolghadri, Ali. Interval state estimation for a class of nonlinear systems. IEEE Transactions on Automatic Control, 57(1):260-265, 2012. DOI: 10.1109/tac.2011.2164820. 
[24] Ríos, Héctor, Mincarelli, Diego, Efimov, Denis, Perruquetti, Wilfrid, and Davila, Jorge. Continuous and discrete state estimation for switched LPV systems using parameter identification. Automatica, 62:139-147, 2015. DOI: 10.1016/j.automatica.2015.09.016.

[25] Robinson, Elinirina I, Marzat, Julien, and Raïssi, Tarek. Interval observer design for unknown input estimation of linear time-invariant discrete-time systems. IFAC-PapersOnLine, 50(1):4021-4026, July 2017, Toulouse, France. DOI: $10.1016 / j$.ifacol.2017.08.717.

[26] Sontag, Eduardo D and Wang, Yuan. On characterizations of the inputto-state stability property. Systems \& Control Letters, 24(5):351-359, 1995. DOI: $10.1016 / 0167-6911$ (94)00050-6.

[27] Thabet, Rihab El Houda, Raïssi, Tarek, Combastel, Christophe, Efimov, Denis, and Zolghadri, Ali. An effective method to interval observer design for time-varying systems. Automatica, 50(10):2677-2684, 2014. DOI: 10.1016/j.automatica.2014.08.035.

[28] Vazquez, Sergio, Rodriguez, Jose, Rivera, Marco, Franquelo, Leopoldo G, and Norambuena, Margarita. Model predictive control for power converters and drives: Advances and trends. IEEE Transactions on Industrial Electronics, 64(2):935-947, 2017. DOI: 10.1109/tie.2016.2625238.

[29] Wang, Yan, Bevly, David M, and Rajamani, Rajesh. Interval observer design for LPV systems with parametric uncertainty. Automatica, 60:79-85, 2015. DOI: $10.1016 / j$.automatica.2015.07.001.

[30] Wang, Zhenhua, Lim, Cheng-Chew, and Shen, Yi. Interval observer design for uncertain discrete-time linear systems. Systems \& Control Letters, 116:41-46, 2018. DOI: $10.1016 / j$.sysconle.2018.04.003.

[31] Zhang, Zhi-Hui and Yang, Guang-Hong. Fault detection for discrete-time LPV systems using interval observers. International Journal of Systems Science, 48(14):2921-2935, 2017. DOI: 10.1080/00207721.2017.1363926. 